\title{
Obituary
}

\section{In Memoriam for M. Flint Beal}

Pre-press 13 August 2021

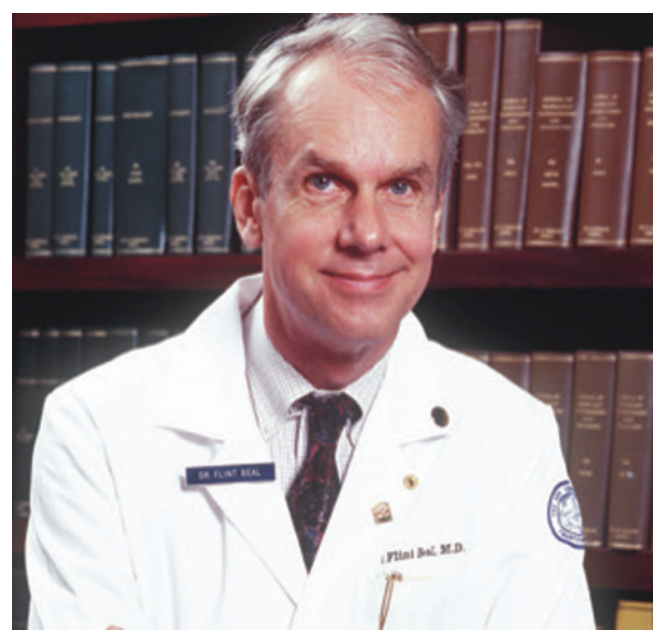

M. Flint Beal, a leading neurologist and translational neuroscientist in the field of neurodegenerative diseases, passed away this June. Flint was an internationally widely-recognized and admired physician-scientist who was dedicated to combining patient care with leading translational research on neurodegenerative diseases.

Flint graduated from medical school in 1976 from the University of Virginia, and after medical internship at New York Hospital-Cornell, he completed his neurology residency at Harvard's Massachusetts General Hospital, where he then rose to head of the Neurochemistry Laboratory and professor of neurology prior to his move back to Cornell in 1998. From 1998 to 2012 he was chairman of the then joint Department of Neurology \& Neuroscience at Weill Cornell, as well as physician-in-chief of neurology at the New York Presbyterian Hospital-Cornell. His clinical subspeciality was in memory disorders. At the time of his death, he was university professor of neurology and neuroscience at the Feil Family Brain and Mind Research Institute of Weill Cornell.

His broad and detailed knowledge allowed him to undertake high level research on several major neurological diseases, including Alzheimer's, Parkinson's, and Huntington's diseases, as well as amyotrophic lateral sclerosis. In his research he focused on the major question of how aging, the most important risk factor for neurodegenerative diseases, could selectively affect vulnerable brains areas and be targeted for treatment by combating oxidative stress and optimizing mitochondrial function. Flint worked mostly in preclinical therapeutic development, using rodent models of disease but also set up experimental clinical trials for patients. His efforts in these important areas of research and therapy will undoubtedly continue. Flint was kind and soft-spoken, and supportive of his trainees, both in the clinic and the laboratory. His encyclopedic memory was widely admired. He could provide details on articles (clinical and research) from decades ago and draw out extended biochemical pathways typically only depicted in textbooks. Flint was highly productive, publishing over 500 articles and 125 chapters, (over 115,000 citations) and was on numerous editorial boards, including Annals of Neurology, Journal of Neurochemistry, and Neurobi- 
ology of Disease. He was a member of the National Academy of Medicine.

Even as head of a department, Flint spent significant time attending scientific and medical conferences, often returning with new ideas for experiments, in particular for potential new experimental therapy studies. At conferences he spent time carefully going over posters and interacting with young scientists, which is not always the case with leading PIs. Flint trained numerous experimental and clinical scientists, and he influenced many others during his long and successful career. He was among a dwindling generation of leading neurologist-scientists with such a broad and comprehensive understanding of diseases of the brain.
Gunnar K. Gouras

Professor of Experimental Neurology

Head, Section of Neurobiology and Experimental

Dementia Research Unit

Department of Experimental Medical Science

Faculty of Medicine, Lund University

E-mail: gunnar.gouras@med.lu.se

\section{ACKNOWLEDGMENTS}

The input from Michael Lin, Magali Dumont, and Davide Tampellini was appreciated. 